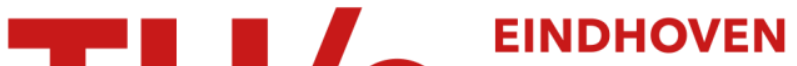

\section{Measures for pathway analysis in brain white matter using diffusion tensor images}

\section{Citation for published version (APA):}

Astola, L., Florack, L., \& Haar Romeny, B. T. (2007). Measures for pathway analysis in brain white matter using diffusion tensor images. In N. Karssemeijer, \& B. Lelieveldt (Eds.), Information Processing in Medical Imaging 20th International Conference, IPMI 2007, Proceedings (pp. 642-649). (Lecture Notes in Computer Science; Vol. 4584). Springer. https://doi.org/10.1007/978-3-540-73273-0_53, https://doi.org/10.1007/978-3-540-73273-0_53

DOI:

10.1007/978-3-540-73273-0 53

10.1007/978-3-540-73273-0_53

Document status and date:

Published: 01/12/2007

\section{Document Version:}

Publisher's PDF, also known as Version of Record (includes final page, issue and volume numbers)

\section{Please check the document version of this publication:}

- A submitted manuscript is the version of the article upon submission and before peer-review. There can be important differences between the submitted version and the official published version of record. People interested in the research are advised to contact the author for the final version of the publication, or visit the $\mathrm{DOI}$ to the publisher's website.

- The final author version and the galley proof are versions of the publication after peer review.

- The final published version features the final layout of the paper including the volume, issue and page numbers.

Link to publication

\section{General rights}

Copyright and moral rights for the publications made accessible in the public portal are retained by the authors and/or other copyright owners and it is a condition of accessing publications that users recognise and abide by the legal requirements associated with these rights.

- Users may download and print one copy of any publication from the public portal for the purpose of private study or research.

- You may not further distribute the material or use it for any profit-making activity or commercial gain

- You may freely distribute the URL identifying the publication in the public portal.

If the publication is distributed under the terms of Article 25fa of the Dutch Copyright Act, indicated by the "Taverne" license above, please follow below link for the End User Agreement:

www.tue.nl/taverne

Take down policy

If you believe that this document breaches copyright please contact us at:

openaccess@tue.nl

providing details and we will investigate your claim. 


\title{
Measures for Pathway Analysis in Brain White Matter Using Diffusion Tensor Images ${ }^{\star}$
}

\author{
Laura Astola, Luc Florack, and Bart ter Haar Romeny \\ Eindhoven University of Technology, PO Box 513, NL-5600 MB Eindhoven, \\ The Netherlands
}

\begin{abstract}
In this paper we discuss new measures for connectivity analysis of brain white matter, using MR diffusion tensor imaging. Our approach is based on Riemannian geometry, the viability of which has been demonstrated by various researchers in foregoing work. In the Riemannian framework bundles of axons are represented by geodesics on the manifold. Here we do not discuss methods to compute these geodesics, nor do we rely on the availability of geodesics. Instead we propose local measures which are directly computable from the local DTI data, and which enable us to preselect viable or exclude uninteresting seed points for the potentially time consuming extraction of geodesics. If geodesics are available, our measures can be readily applied to these as well.

We consider two types of geodesic measures. One pertains to the connectivity saliency of a geodesic, the second to its stability with respect to local spatial perturbations. For the first type of measure we consider both differential as well as integral measures for characterizing a geodesic's saliency either locally or globally. (In the latter case one needs to be in possession of the geodesic curve, in the former case a single tangent vector suffices.) The second type of measure is intrinsically local, and turns out to be related to a well known tensor in Riemannian geometry.
\end{abstract}

Keywords: DTI, geodesics, brain white matter connectivity, geodesic deviation, Riemann tensor, Ricci tensor.

\section{Introduction}

The traditional MR-DTI data matrix gives a Gaussian probabilistic model for the diffusion of water molecules in six different directions in each volume element of a 3D image. If diffusivity is large in a certain direction, then the time a water molecule travels a given distance in this direction is short. Thus the positive definite data matrix can be interpreted as reciprocally proportional to a Riemannian metric tensor as is pointed out by O'Donnell et al. 11 and Lenglet et al. 2]. Having a metric tensor attached to each point (on a compact manifold), we can solve for the shortest path, i.e. a minimal geodesic between a given

\footnotetext{
* The Netherlands Organisation for Scientific Research (NWO) is gratefully acknowledged for financial support.
} 
pair of points. For this purpose, there are various numerical methods ranging from techniques similar to Dijkstra's algorithm [345], level set methods [26], to solving the discretized Hamilton-Jacobi equation of propagating wavefronts [7].

Whatever the method for computing geodesics, one has to appreciate that although a coherent bundle of axons with resolvable thickness is likely to produce geodesics in (uncorrupted) DTI data, an arbitrarily chosen geodesic is unlikely to correspond to a bundle of axons. (Geodesics "run all over the place", so to speak.) Therefore, we propose a set of measures for the connection strength of a geodesic, both locally as well as globally (Section 2), and for its sensitivity to small spatial perturbations of the initial seed point (Section [3). A large connection strength implies that a candidate geodesic is more likely to correspond to an actual axon bundle (or other physical water channel). The stability measure, on the other hand, quantifies the amount of deviation from nearby geodesics, akin to the relative acceleration of freely falling particles in an inhomogeneous gravitational field (metric). One expects this deviation to be small in fibrous tissue in which the fibres are well aligned, and large in chaotic regions.

In short, we answer the question whether and to which extent a geodesic can be seen as a representative member of an articulated, coherent bundle of neighbouring geodesics, and which points are (un)likely to be part of such a structure.

\section{Geodesics Versus DTI Fibres}

In white matter microstructures inhibit the free Brownian motion of water molecules. the myelinated neurons in brain white matter favor diffusion along and impede diffusion across their tangent directions. Therefore, along a meaningful bundle of axons, the diffusivity is relatively large.

In the following we use Einstein summation convention: $a_{i} b^{i} \equiv \sum_{i=1}^{n} a_{i} b^{i}$. On a compact simply connected manifold, every pair of points can be connected by a geodesic, i.e. a curve of extremal energy (with affine parameter $t$ )

$$
E(\gamma)=\frac{1}{2} \int_{0}^{T} g_{i j}(\gamma(t)) \dot{\gamma}^{i}(t) \dot{\gamma}^{j}(t) d t,
$$

or equivalently, of the (parametrization independent) length functional

$$
L(\gamma)=\int_{0}^{T} \sqrt{g_{i j}(\gamma(t)) \dot{\gamma}^{i}(t) \dot{\gamma}^{j}(t)} d t
$$

Here we want to measure the quality of a geodesic as a carrier of diffusion, i.e. how likely it is for the geodesic to actually be generated by a bundle of fibres (or other physical water channel). As a measure of the relative diffusivity along a geodesic we take the ratio of lengths or energies given by the Euclidean and diffusion induced Riemannian metric tensors, respectively. Let $g=D^{-1}$, where $D$ is the DTI matrix field, with components $g_{i j}$ relative to a coordinate basis: $g(x)=g_{i j}(x) d x^{i} \otimes d x^{j}$, and let $\gamma(t)$ be a parameterized geodesic for the metric 
$g$, starting at point $p=\gamma(0)$, with tangent $\dot{\gamma}(t)$. The proposed measures are then given by

$$
m_{E}(\gamma)=\frac{\int_{0}^{T} \delta_{i j} \dot{\gamma}^{i}(t) \dot{\gamma}^{j}(t) d t}{\int_{0}^{T} g_{k l}(\gamma(t)) \dot{\gamma}^{k}(t) \dot{\gamma}^{l}(t) d t} \quad \text { resp. } \quad m_{L}(\gamma)=\frac{\int_{0}^{T} \sqrt{\delta_{i j} \dot{\gamma}^{i}(t) \dot{\gamma}^{j}(t)} d t}{\int_{0}^{T} \sqrt{g_{k l}(\gamma(t)) \dot{\gamma}^{k}(t) \dot{\gamma}^{l}(t)} d t} .
$$

It should be stressed that neither equals the so-called validity index, cf. [7].

In the neighbourhood of point $p$ on $\gamma$, the limit of each ratio in Eq. (3) for $T \rightarrow 0$ gives us a local measure of the connection strength:

$$
m_{E}(V)=\frac{\delta_{i j} V^{i} V^{j}}{g_{k l}(p) V^{k} V^{l}} \quad \text { resp. } \quad m_{L}(V)=\frac{\sqrt{\delta_{i j} V^{i} V^{j}}}{\sqrt{g_{k l}(p) V^{k} V^{l}}}
$$

where $V=\dot{\gamma}(0)$.

If we denote by $V^{+} \equiv \operatorname{argmax}(m(V))$ the principal eigenvector of $D=g^{-1}$ with eigenvalue $\lambda_{+}$, say, then

$$
m_{E}\left(V^{+}\right)=\lambda_{+} \quad \text { resp. } \quad m_{L}\left(V^{+}\right)=\sqrt{\lambda_{+}}
$$

are indeed (up to monotonic transformations) the most reasonable a priori local measures.

Thus locally, in anisotropic voxels, our measure gives maxima in the direction of the eigenvector that corresponds to the largest eigenvalue of the DTI-tensor, and coincides with traditional largest eigenvalue fibre tracking. However, by splitting up the integrals in (3) over a partitioning of the curve $\gamma$ into subcurves $\gamma_{\alpha}$, we may apply the integral measures to any curve segment $\gamma_{\alpha}$, and measure possible variations in diffusivity along the curve to any desired level of discretization. In this way we obtain a set of integral connectivity measures for an arbitrary partitioning of a given (not necessarily geodesic) curve. If $\gamma=\cup_{\alpha=1}^{N} \gamma_{\alpha}$ is a partitioning of a curve $\gamma:[0, T] \rightarrow \mathbb{R}^{3}$ into any number $N$ of curve segments $\gamma_{\alpha}:\left[t_{\alpha-1}, t_{\alpha}\right] \rightarrow \mathbb{R}^{3}$ (with $t_{0}=0, t_{N}=T$ ), then from Eq. (3) we obtain an arbitrarily large set of submeasures,

$$
m_{E}^{(\alpha)}=m_{E}\left(\gamma_{\alpha}\right) \quad \text { resp. } \quad m_{L}^{(\alpha)}=m_{L}\left(\gamma_{\alpha}\right)
$$

If a curve (segment) $\gamma$ corresponds to an actual fibre bundle, then both $m_{E}(\gamma)$ and $m_{L}(\gamma)$ will be large, since the denominator will be small. Note also that the magnitudes of $m_{L, E}(\gamma)$ are not biased w.r.t. the length of the curve (unlike numerator and denominator separately, which do scale with curve length, cf. the similar but non-invariant measure proposed by Prados [8]). This motivates our choice for Eq. (3).

\section{Geodesic Deviation}

The concept of geodesic deviation pertains to the relative acceleration by which two hypothetical test particles in "free fall" along two neighbouring geodesics 

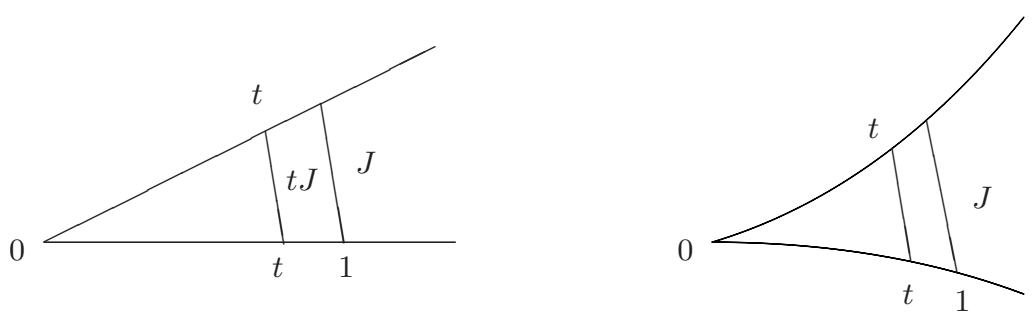

Fig. 1. Left: $\ddot{J}=0$ (no geodesic deviation). Right: $\ddot{J}>0$ as a result of curvature.

separate. That is, if one moves along two geodesics that start out from the same seed point at $t=0$, say, with initial velocity vectors of equal magnitude, differing only in relative directions, one naturally observes that their mutual distance increases. This geodesic separation as such is trivial. In a flat space it is linear in $t$, so that the relative acceleration between the two points vanishes identically. However, in a curved space this is different (just think of the great circles of a sphere, which are geodesics if one is confined to its curved surface, cf. Fig. 1). In general there is a deviation from the lowest order linear behaviour, which will (initially) cause either an acceleration or deceleration in the mutual separation of the points. One can show that, if $J(t)$ denotes the separation vector 11 connecting any point $\gamma(t)$ on a fiducial geodesic at time $t$ to a corresponding one on a neighbouring geodesic indicated by the relative separation vector $J(t)$, then

$$
\frac{D^{2} J(t)}{d t^{2}}+R\left(\gamma^{\prime}(t), J(t)\right) \gamma^{\prime}(t)=0
$$

The symbol $D$ denotes covariant derivative, and $R$ is the so-called Riemann curvature. One can show that the magnitude of the relative separation vector $J(t)$ initially evolves as

$$
|J(t)|=t-\frac{1}{6}\langle R(V, W) V, W\rangle t^{3}+\mathcal{O}\left(t^{4}\right)
$$

in which $V=\gamma^{\prime}(0)$ and $W=J^{\prime}(0)$. Thus the interesting quantity is the coefficient of the $\mathcal{O}\left(t^{3}\right)$ term, which contains all relevant curvature information responsible for geodesic deviation.

In a local coordinate system Eq. (7) becomes

$$
\frac{D^{2} J^{i}(t)}{d t^{2}}+R_{j k l}^{i}(\gamma(t)) \frac{d \gamma^{j}(t)}{d t} J^{k}(t) \frac{d \gamma^{l}(t)}{d t}=0 .
$$

\footnotetext{
$\overline{{ }^{1} \text { Formally } J}(t)$ is defined in terms of the exponential map, $J(t)=\left(d \exp _{p}\right)_{t V} t W$, in which $p$ is the base point of interest, $V$ is a tangent to a fiducial geodesic, and $W$ a second tangent which "selects" a neighbouring geodesic. We refer to the literature for details.
} 
Following do Carmo's index convention [9], the Riemann curvature tensorsecond term in Eq. (77) - is defined as

$$
R(X, Y) Z=R_{i j k}^{l} X^{i} Y^{j} Z^{k} \frac{\partial}{\partial x_{l}}
$$

in terms of its components, which are given by the functions (spatial arguments $x \in \mathbb{R}^{3}$ are implicit)

$$
R_{i j k}^{m}=\Gamma_{i k}^{l} \Gamma_{j l}^{m}-\Gamma_{j k}^{l} \Gamma_{i l}^{m}+\frac{\partial}{\partial x_{j}} \Gamma_{i k}^{m}-\frac{\partial}{\partial x_{i}} \Gamma_{j k}^{m},
$$

in which the Christoffel symbols are defined as

$$
\Gamma_{i j}^{k}=\frac{1}{2} g^{k l}\left\{\frac{\partial g_{j l}}{\partial x^{i}}+\frac{\partial g_{l i}}{\partial x^{j}}-\frac{\partial g_{i j}}{\partial x^{l}}\right\} .
$$

(Recall that $g^{i j}(x)$ are just the entries of the DTI matrix at point $x$, and $g_{i j}(x)$ the entries of its inverse.)

Although a detailed explanation of this geometric analysis is far beyond the scope of this paper, it suffices to appreciate the heuristics of our approach. The interested reader is referred to do Carmo [9] or any other suitable text book on Riemannian geometry for further details and proofs.

In this section, our next goal is to obtain a measure for geodesic deviation that (i) is a purely local entity, and (ii) involves only the geodesic direction (i.e. $\left.V=\gamma^{\prime}(0)\right)$ and does not prefer any extrinsically chosen plane through $V$, in other words, does not contain the vector $W=J^{\prime}(0)$. To this end we may average the relevant coefficient of Eq. (8) over all independent vectors $W_{a}, a=1, \ldots, n-1$, perpendicular to $V=\gamma^{\prime}(0)$. In this way one obtains the so-called Ricci curvature:

$$
\operatorname{Ric}_{p}(V)=\frac{1}{n-1} \sum_{a=1}^{n-1}\left\langle R\left(V, W_{a}\right) V, W_{a}\right\rangle .
$$

In particular, in the 3-dimensional case at hand, we have two mutually orthonormal vectors $W_{1}, W_{2} \perp V$, and we may obtain the average as:

$$
\begin{aligned}
\operatorname{Ric}_{p}(V) & =\frac{1}{2 \pi} \int_{0}^{2 \pi}\left\langle R\left(V, \cos \theta W_{1}+\sin \theta W_{2}\right) V, \cos \theta W_{1}+\sin \theta W_{2}\right\rangle d \theta \\
& =\frac{1}{2} \sum_{a=1}^{2}\left\langle R\left(V, W_{a}\right) V, W_{a}\right\rangle,
\end{aligned}
$$

which indeed agrees with the general definition, Eq. (13). Although the r.h.s. still contains the basis $\left\{W_{a}\right\}_{a=1}^{n-1}$ that spans the plane orthogonal to $V$, the result must be independent of its actual choice. To see that this is indeed the case, 
substitute the coordinate expressions of all geometric quantities involved into Eq. (13), using $V=V^{i} \frac{\partial}{\partial x_{i}}, W_{a}=W_{a}^{i} \frac{\partial}{\partial x_{i}}$, and Eq. (10). We obtain

$$
\begin{aligned}
\operatorname{Ric}_{p}(V) & =\frac{1}{n-1} \sum_{a=1}^{n-1}\left\langle R_{i j k}^{l} V^{i} W_{a}^{j} V^{k} \frac{\partial}{\partial x_{l}}, W_{a}^{m} \frac{\partial}{\partial x_{m}}\right\rangle \\
& =\frac{1}{n-1} \sum_{a=1}^{n-1} R_{i j k}^{l} V^{i} V^{k} W_{a}^{m} W_{a}^{j} g_{l m} \\
& =\frac{1}{n-1} \sum_{a=1}^{n-1} R_{i j k m} V^{i} V^{k} W_{a}^{m} W_{a}^{j}
\end{aligned}
$$

in which we have defined $R_{i j k m}=g_{l m} R_{i j k}^{l}$. In $n$ dimensions $\left\{W_{1}, W_{2}, \ldots\right.$, $\left.W_{n-1}, V\right\}$ constitutes an orthonormal basis, as a result of which we have

$$
\sum_{a=1}^{n-1} W_{a}^{j} W_{a}^{m}=g^{j m}-V^{j} V^{m} .
$$

Substitution into Eq. (16) yields, abbreviating $R_{i k}=g^{m j} R_{i j k m}$,

$$
\begin{aligned}
\operatorname{Ric}_{p}(V) & =\frac{1}{n-1} R_{i j k m} V^{i} V^{k}\left(g^{j m}-V^{j} V^{m}\right) \\
& =\frac{1}{n-1} R_{i k} V^{i} V^{k}-\frac{1}{n-1} \sum_{h=1}^{n-1} R_{i j k m} V^{i} V^{k} V^{j} V^{m}
\end{aligned}
$$

By virtue of the symmetries of the Riemann tensor the last summand vanishes, and so we end up with

$$
\operatorname{Ric}_{p}(V)=\frac{1}{n-1} R_{i j} V^{i} V^{j}
$$

Eq. (18) is our main result with respect to geodesic deviation. (In our case, $n=3$, but the proportionality factor is immaterial.) Note that it satisfies our previous requirements, i.e. it depends only on differential DTI properties, and only involves a directional argument. Thus one does not need to know the geodesics in order to compute geodesic deviation. This greatly facilitates the computation. In fact, given a unit tangent vector $V$, Eq. (18) can be obtained by a somewhat lengthy but completely straightforward algebraic combination of partial derivatives of the DTI image of orders $0,1,2$. The algorithm is as follows:

1. Compute the metric $g_{i j}$ by pointwise inversion of the DTI matrix $g^{i j}$.

2. Compute the Christoffel symbols $\Gamma_{i j}^{k}$, Eq. (12).

3. Compute the components of the Riemann tensor $R_{i j k}^{l}$, Eq. (11).

4. Obtain the Ricci tensor by contraction: $R_{i j}=R_{i j k}^{k}$.

5. Specify the components of a unit tangent $V^{i}$, and contract onto the Ricci tensor so as to obtain the proposed measure for geodesic deviation: $R_{i j} V^{i} V^{j}$. 
The second and third steps require the computation of derivatives up to second order. This can e.g. be done in the usual way within the framework of scale space theory, taking into account a scale parameter that admits a sufficiently accurate extraction of second order derivatives (a Gaussian scale slightly larger than one voxel may already yield acceptable results depending on noise level, cf. [10]1]). The algorithm may be augmented with a scale selection procedure for optimal performance, since the "right" scale for pathway analysis is not known a priori. This highly interesting but nontrivial option is not further pursued here.

\section{Conclusions}

We have proposed two different types of differential geometric measures for DTI pathway analysis, and operational schemes to compute them. The first one, the connection strength, is a zeroth order differential property that gives information about the relative diffusivity along a given curve. Curves with large connection strengths are more likely to correspond to actual elongated structures of axons or other physical water channels. Our connectivity measure can be applied to any not necessarily geodesic curve, to an arbitrary segment and, in the limit, even to any point of such a curve. For the preselection of viable seed points for geodesics, it is, apart from quantifying their connectivity, likewise useful to compute their geodesic deviation as a measure for local stability and coherence. We have argued that the Ricci curvature in the direction of largest diffusion is an appropriate measure for this. A positive Ricci curvature, which depends on second order derivatives of the DTI image, indicates the presence of bundles of geodesics that are well aligned. Combination of the two types of measures can be used to single out suitable seed points for, and to judge the saliency of, a fiducial geodesic.

\section{References}

1. O'Donnell, L., Haker, S., Westin, C.F.: New approaches to estimation of white matter connectivity in diffusion tensor mri: Elliptic PDEs and geodesics in a tensorwarped space. In: Dohi, T., Kikinis, R. (eds.) MICCAI 2002. LNCS, vol. 2488, pp. 459-466. Springer, Heidelberg (2002)

2. Lenglet, C., Deriche, R., Faugeras, O.: Inferring white matter geometry from diffusion tensor MRI: Application to connectivity mapping. In: Pajdla, T., Matas, J. (eds.) ECCV 2004. LNCS, vol. 3024, pp. 127-140. Springer, Heidelberg (2004)

3. Dijkstra, E.W.: A note on two problems in connexion with graphs. Numerische Mathematik 1(1), 267-271 (1959)

4. Kimmel, R., Sethian, J.A.: Computing geodesic paths on manifolds. In: Proceedings of the National Academy of Sciences, vol. 95, pp. 8431-8435 (1998)

5. Tsitsiklis, J.N.: Efficient algorithms for globally optimal trajectories. IEEE Transactions on Automatic Control 40(9), 1528-1538 (1995)

6. Osher, S., Sethian, J.A.: Fronts propagating with curvature-dependent speed: Algorithms based on Hamilton-Jacobi formulations. Journal of Computational Physics 79, 12-49 (1988) 
7. Jackowski, M., Kao, C.Y., Qiu, M., Costable, R.T., Staib, L.H.: White matter tractography by anisotropic wavefront evolution and diffusion tensor imaging. Medical Image Analysis 9, 427-440 (2005)

8. Prados, E., Soatto, S., Lenglet, C., Pons, J.P., Wotawa, N., Deriche, R., Faugeras, O.: Control theory and fast marching techniques for brain connectivity mapping. In: Proceedings of the IEEE Computer Society Conference on Computer Vision and Pattern Recognition, New York, USA, June 2006, vol. 1, pp. 1076-1083. IEEE Computer Society Press, New York, USA (2006)

9. Carmo, M.P.d.: Riemannian Geometry. Second edn. Mathematics: Theory \& Applications. Birkhäuser, Boston (1993)

10. Blom, J., Haar Romeny, B.M.t., Bel, A., Koenderink, J.J.: Spatial derivatives and the propagation of noise in Gaussian scale-space. Journal of Visual Communication and Image Representation 4(1), 1-13 (1993)

11. Florack, L.M.J., Duits, R.: Regularity classes for locally orderless images. In: Griffin, L.D, Lillholm, M. (eds.) Scale Space Methods in Computer Vision. LNCS, vol. 2695, pp. 255-265. Springer, Heidelberg (2003) 